\title{
Liquid crystalline DNA doped with mini gold nanorods
}

Dominika Benkowska-Biernacka*, Nina Tarnowicz-Staniak, Katarzyna Matczyszyn

Advanced Materials Engineering and Modelling Group, Faculty of Chemistry, Wroclaw University of Science and Technology

* Corresponding author: dominika.benkowska@pwr.edu.pl

Wrocław University of Science and Technology

\section{Faculty of Chemistry}


Abstract: Lyotropic liquid crystals (LCS) seem to play a significant role in biological systems. The well-known example of this material is DNA, that tends to selforganize into various lyotropic mesophases at high concentration water solutions. Previous studies indicated that gold nanoparticles show different arrangement in DNA LCs. It was observed that elongated nanoparticles accumulate in the whole volume of the mesophase, while nanoclusters tend to aggregate on the top of the mesophase.

In the present work, mini gold nanorods (GNRs) have been chosen due to their strong photothermal efficiency. The formation of DNA LCs doped with mini GNRs was investigated and compared with pure DNA LCs by polarized light microscopy. Furthermore, two-photon microscopy was used to image the distribution of mini GNRs in the DNA LC matrix. These systems could potentially be useful as models of interactions of NRs with DNA for biomedical applications such as cancer thermotherapy, owing to the large absorption cross-sections of mini GNRs. Moreover, mini GNRs exhibit higher cellular uptake (especially in tumor changed tissues) and lower cytotoxicity both in vitro and in vivo. Reliable and reproducible protocols describing the synthesis of mini GNRs with well-defined plasmonic and optical properties predestine them as model materials for such investigations.

Keywords: lyotropic liquid crystals; liquid crystalline DNA; gold nanorods; anisotropic gold nanoparticles 


\section{Examples of lyotropic liquid crystals in biological systems}
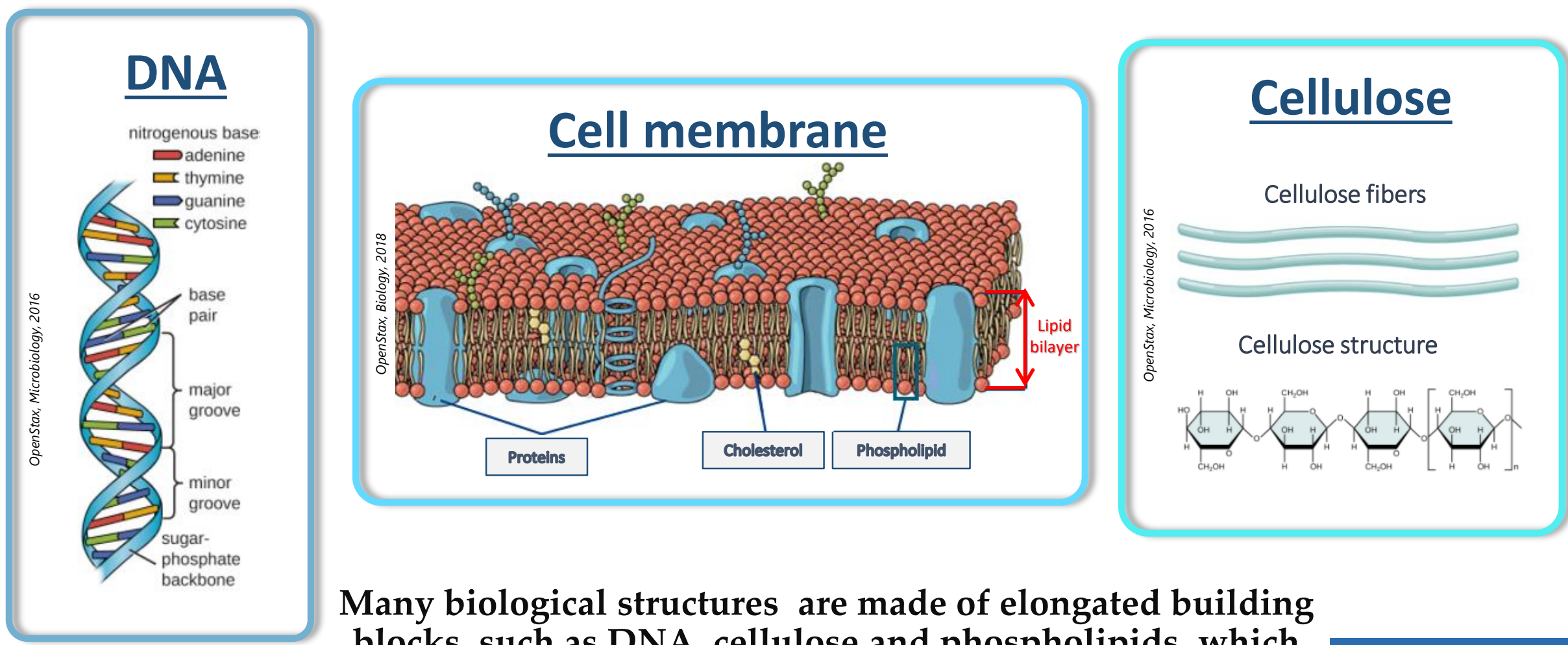

Many biological structures are made of elongated building blocks, such as DNA, cellulose and phospholipids, which are able to self-organize in liquid crystalline phases.

M. Milov, Cholesteric liquid crystals in living matter, Soft Matter, 13, 417 (2017)

Graphics: OpenStax, Biology, 2018 and OpenStax, Microbiology, 2016

Crystals 


\section{What has been done so far}

Liquid crystalline DNA doped with silver-doped gold nanocluster

$=$ AuAg NANOCLUSTERS

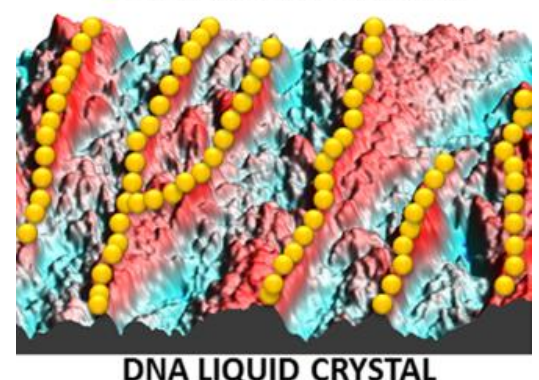

Nanoclusters tend to aggregate on the top of the mesophase.
DNA LIQUID CRYSTAL

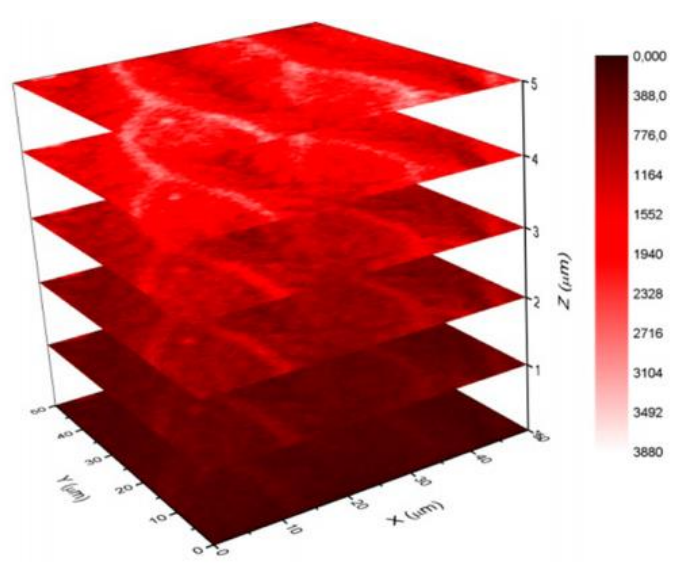

Liquid crystalline DNA doped with gold nanorods ( length of $36 \mathrm{~nm}$ )

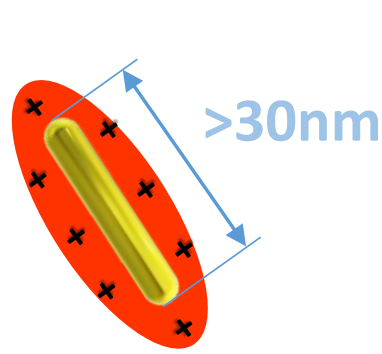

\section{Elongated gold} nanoparticles can accumulate in the whole volume of the mesophase.

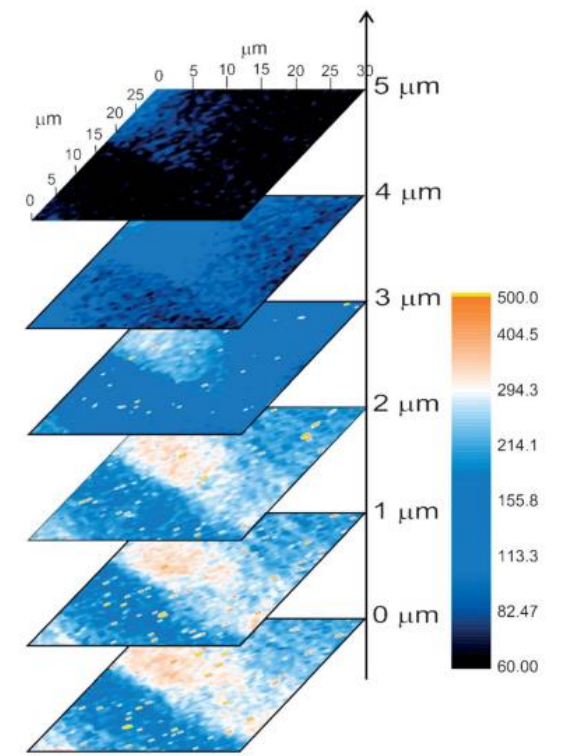

J. Olesiak-Banska, M. Gordel, K. Matczyszyn, V. Shynkar, J. Zyss, M. Samoc, , Langmuir, Nanoscale, 2013, 5, 10975
K. Brach, M. Waszkielewicz, J. Olesiak-Banska, M. Samoc, K. Matczyszyn, Langmuir, 2017, 33, 8993-8999
Crystals 2020 


\section{Materials and Methods}

\section{Mini gold nanorods (GNRs)}

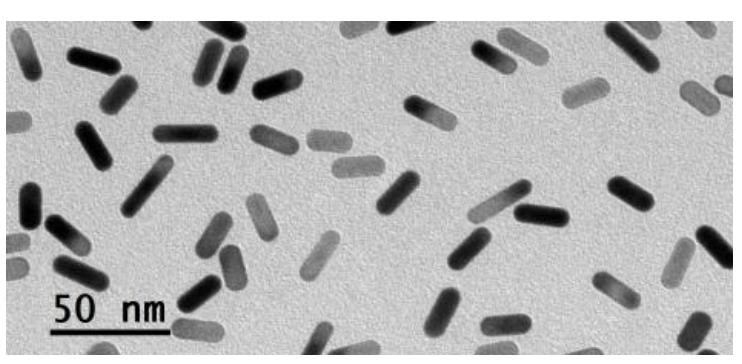

TEM image of mini GNRs

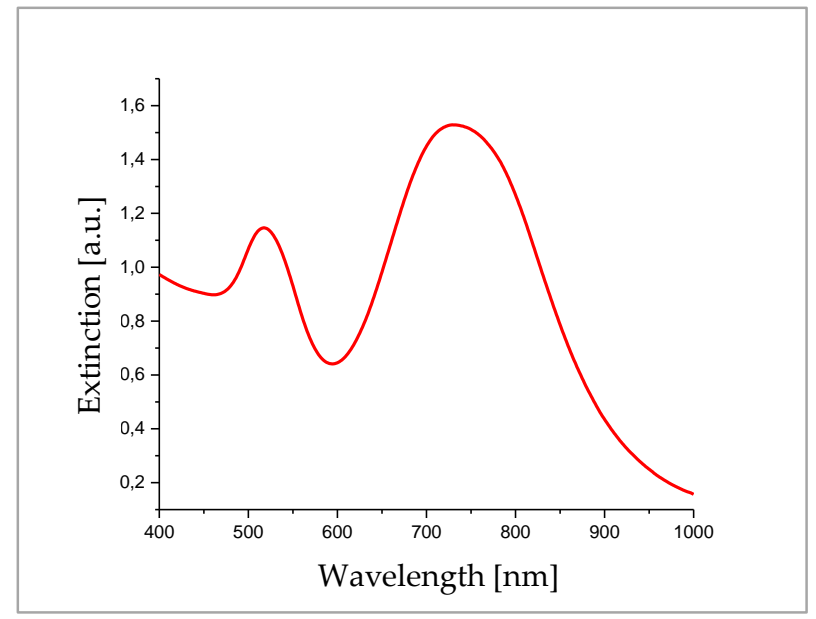

Extinction spectra of mini GNRs
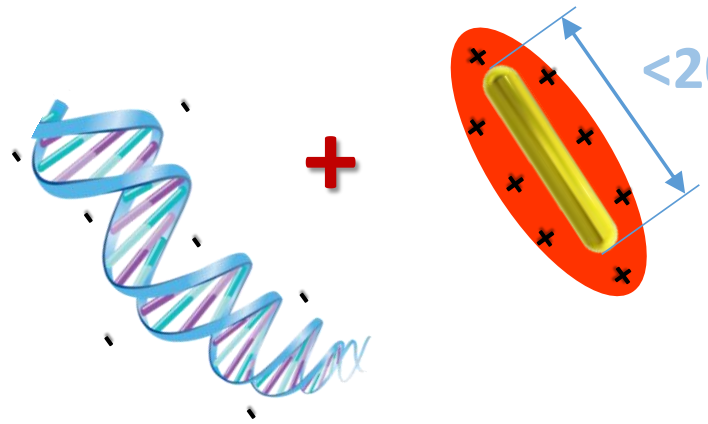

Drying droplet experiment

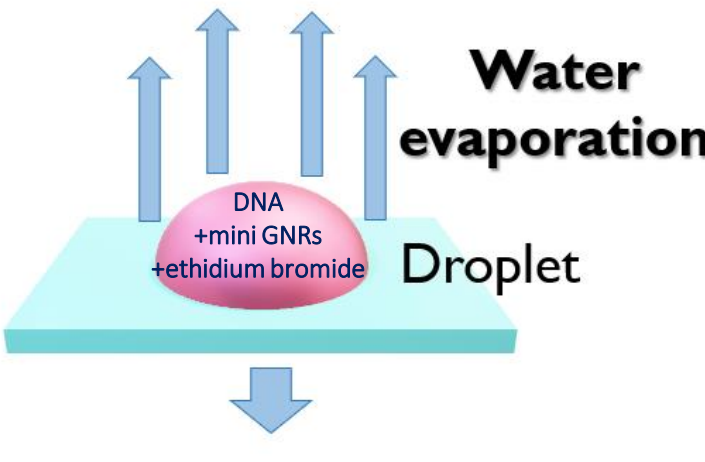

Dried droplet

DNA molecules

in concentrated water solution tend to self-organize into a hexagonal columnar phase on the edge of the droplet.

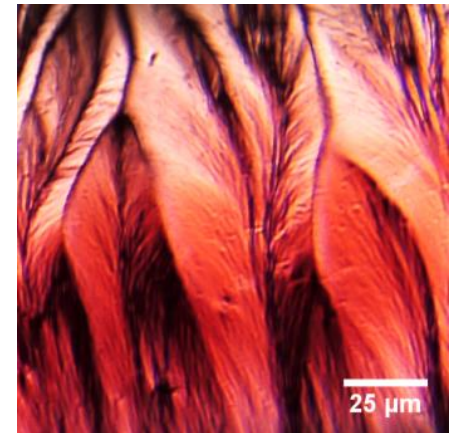

Zig-zag pattern of dried droplet of mini GNRs doped- DNA intercalated with ethidium bromide. The image was taken by polarized light microscope. 


\section{Materials and Methods}

\section{Two-photon fluorescence microscopy setup}

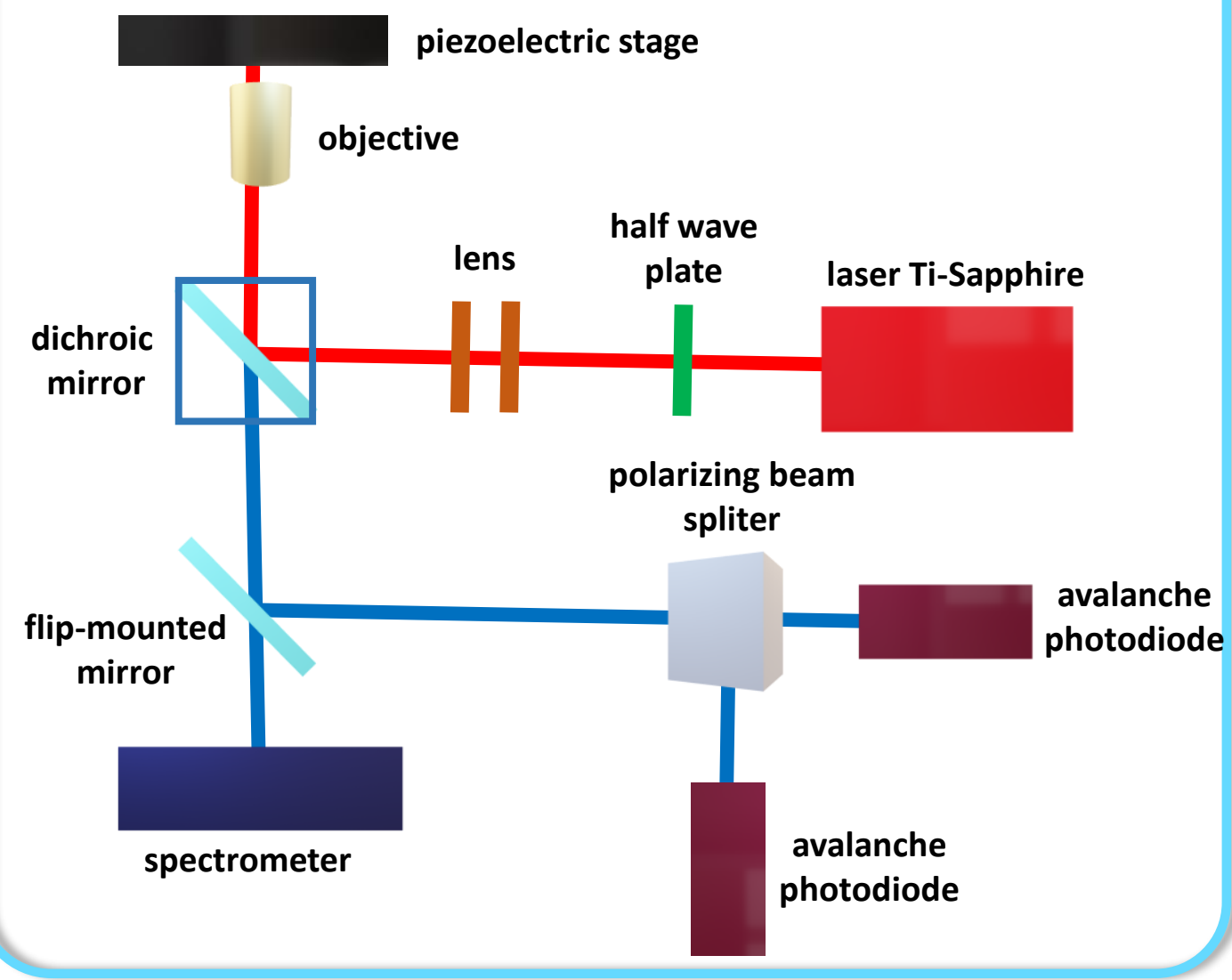

\section{Mini GNRs show two-photon excited luminescence (2PL) emission in liquid crystalline DNA}

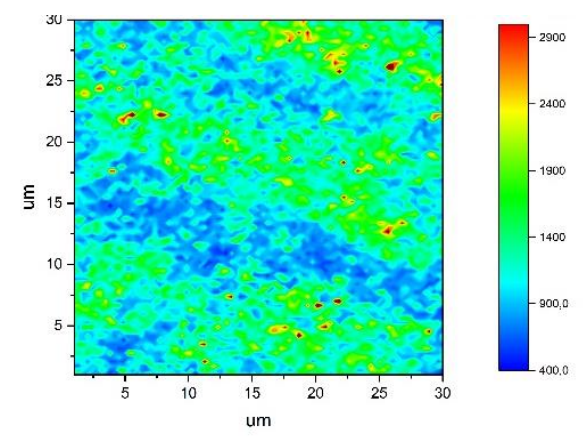

2PL intensity scan of hexagonal columnar phase of DNA liquid crystal doped with gold nanoparticles.

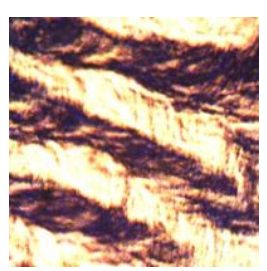

Polarized light microscopy image of the same place of the hexagonal columnar phase of DNA doped with GNRs

\section{Crystals 2020}




\section{Results and discussion}

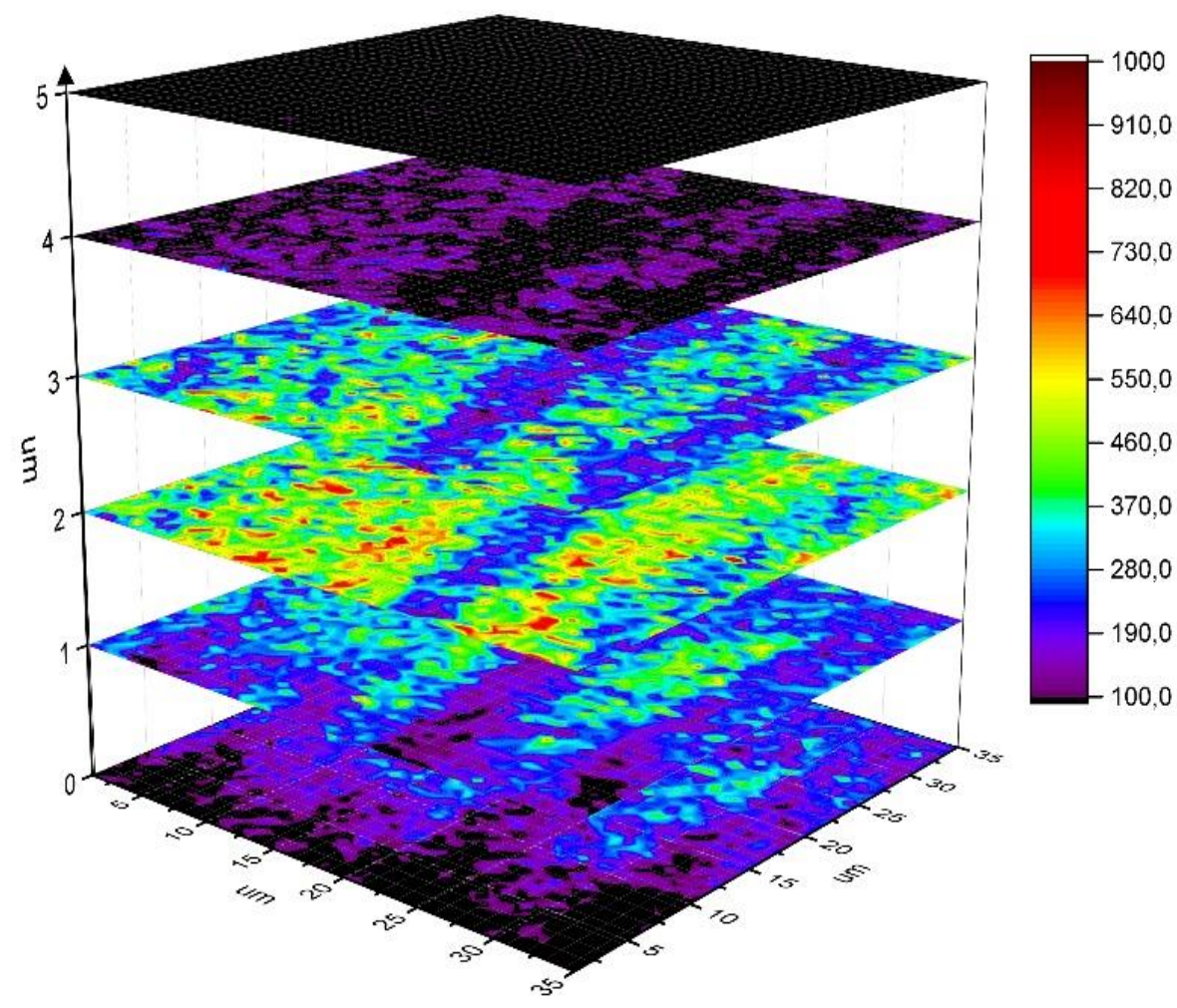

Mini GNRs spread homogenously in the whole volume of the columnar hexagoal phase.

$2 \mathrm{PL}$ intensity scans performed at various depths of hexagonal columnar phase of DNA liquid crystal doped with mini GNRs.

\section{Crystals}




\section{Results and discussion}

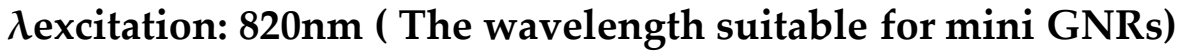

Polarization $45^{\circ}$

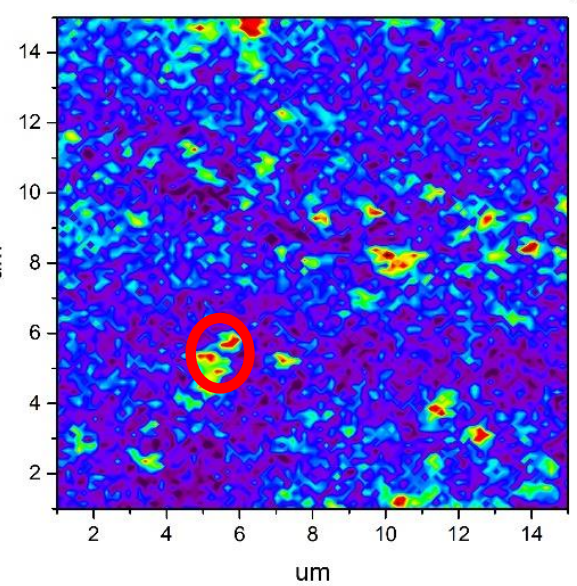

ex820 z18 pol45

Aexcitation: 910nm ( The wavelength suitable for ethidium bromide)

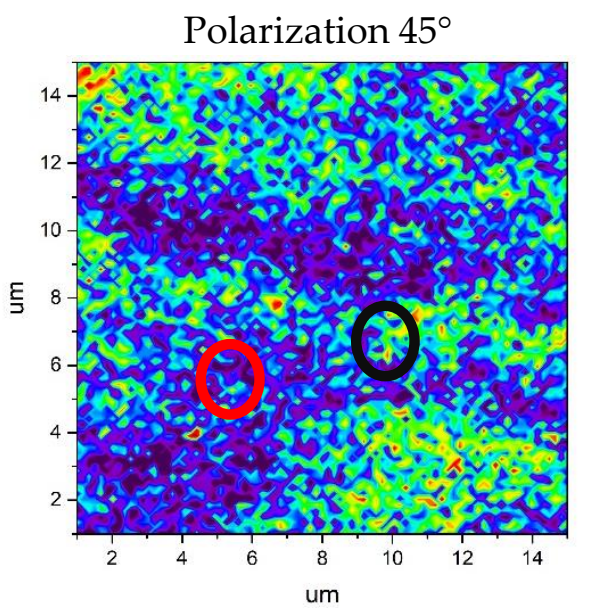

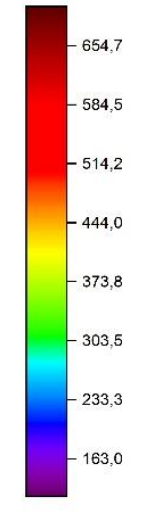

Polarization $-45^{\circ}$

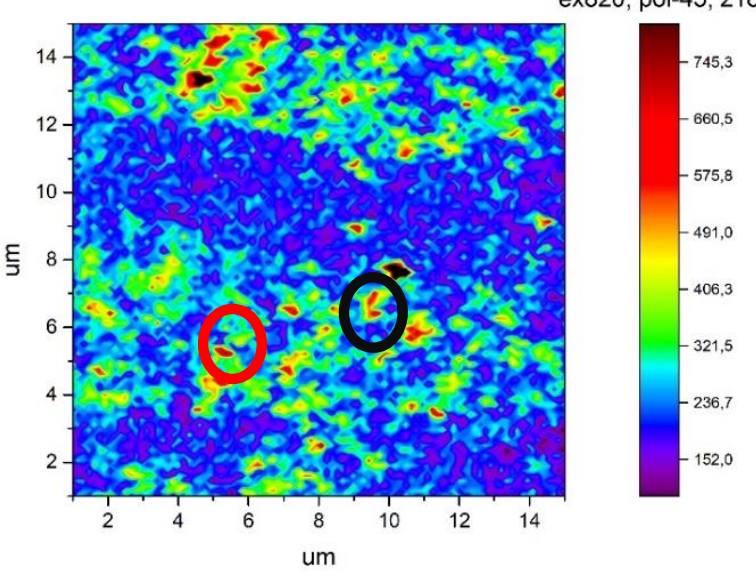

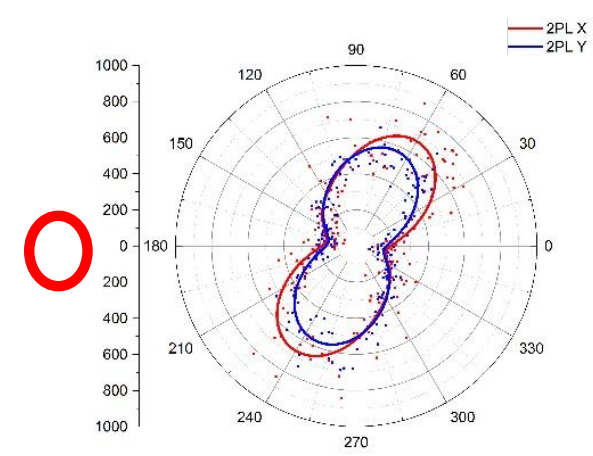

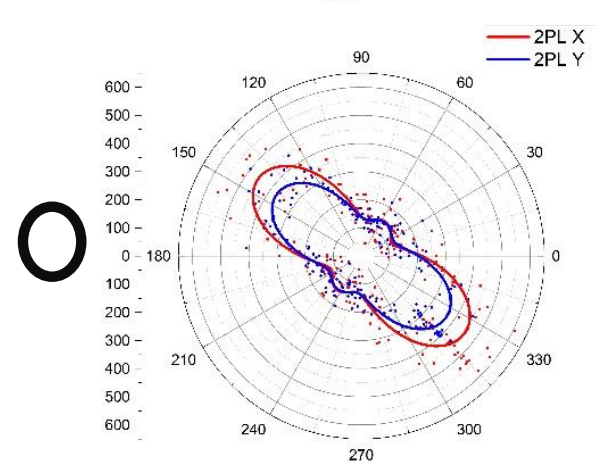

Polar graphs of 2PF of mini GNRs taken from spots marked with red and black circles.

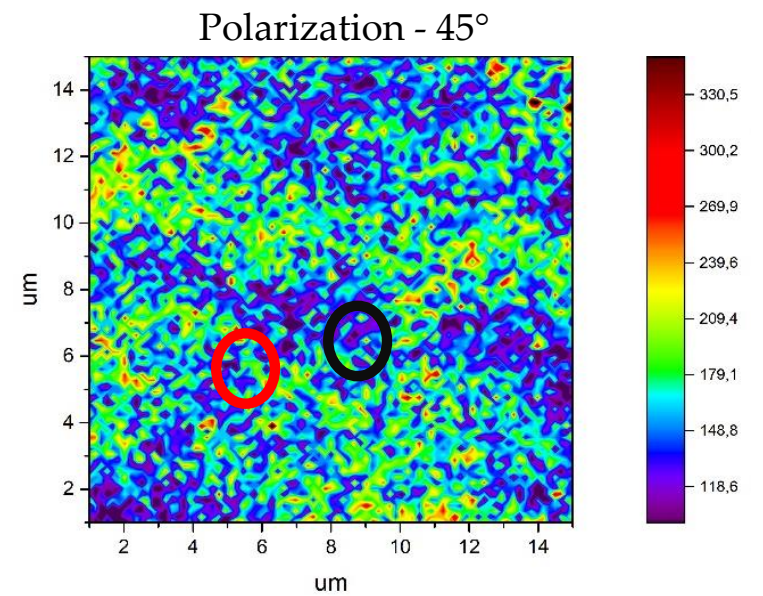

Most of the mini GNRs show tendency to follow the orientation of the long axis of DNA strands. 


\section{Conlusions}

$\checkmark$ Zig-zag pattern of dried droplet of DNA occurs in samples with and without mini GNRs

$\checkmark$ Mini GNRs tend to accumulate within the volume of the columnar hexagonal phase

$\checkmark$ Most of the mini GNRs show tendency to follow orientation of the long axis of DNA strands

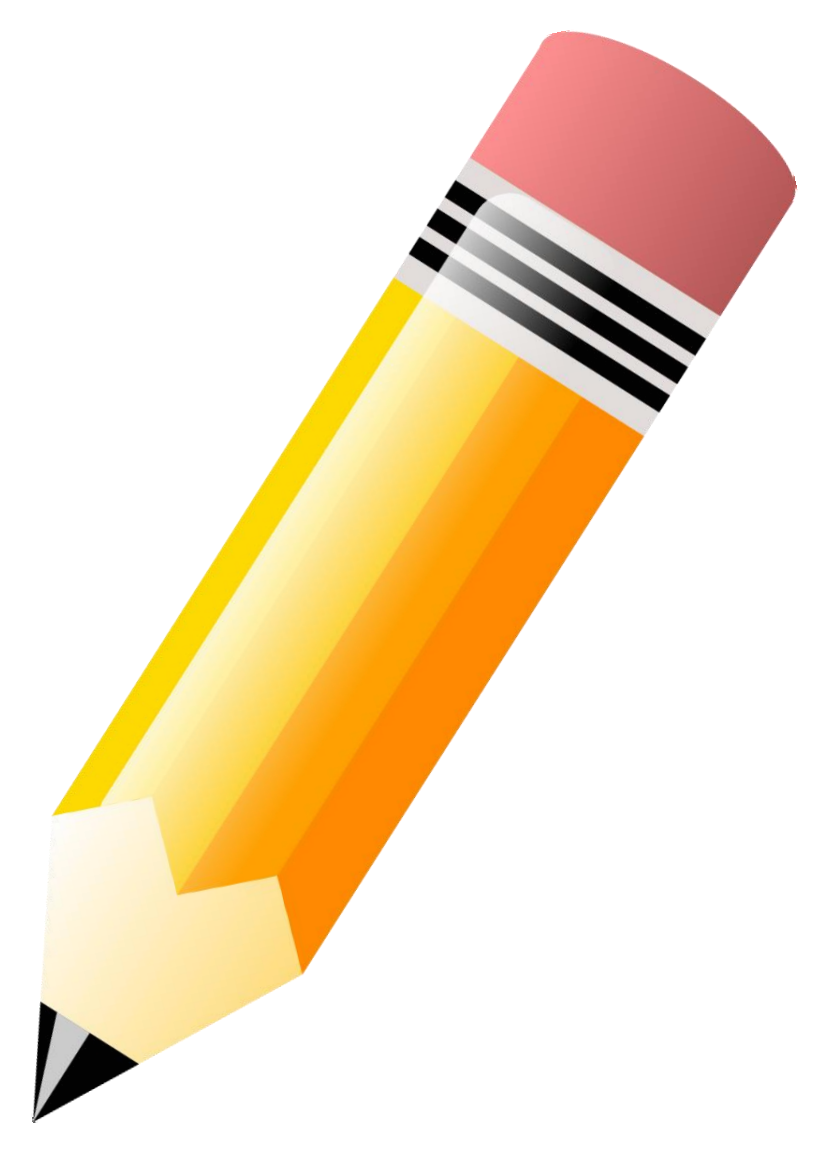

Crystals

2020 


\section{Acknowledgments}

Advanced Materials Engineering and Modelling Group

Wroclaw University of Science and Technology

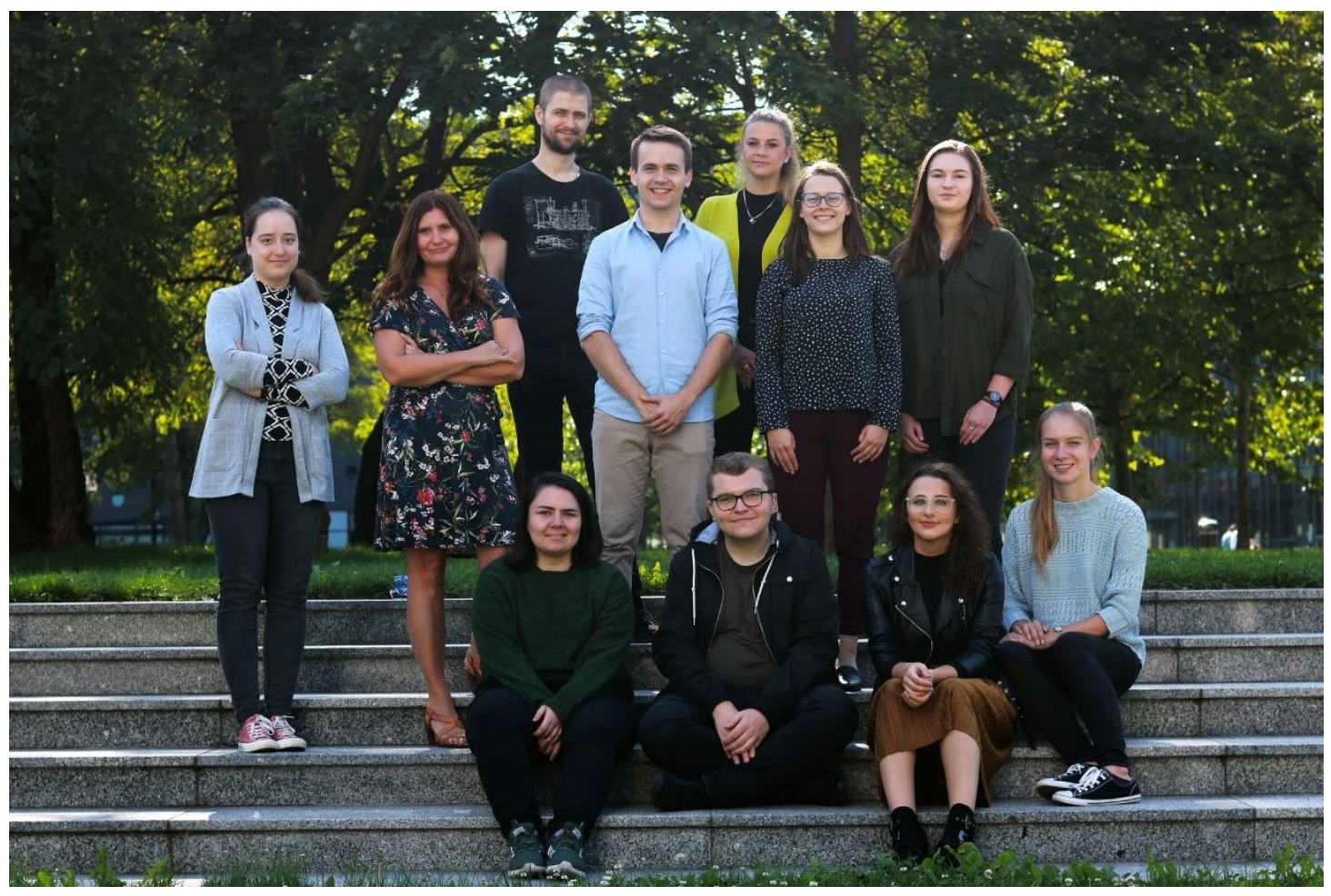

Funded is provided by BioTechNan Programme

European Union European Social Fund

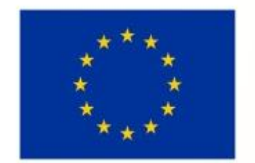

European Funds

Knowledge Education Development

\section{Crystals 2020}

D. Benkowska-Biernacka, N. Tarnowicz-Staniak, K. Matczyszyn - Liquid crystalline DNA doped with mini gold nanorods 